\title{
Synthesis and characterization of superparamagnetic iron-oxide nanoparticles (SPIONs) and utilization of SPIONs in X-ray imaging
}

\author{
C. Justin ${ }^{1} \cdot$ Sheryl Ann Philip ${ }^{1} \cdot$ Antony V. Samrot ${ }^{1}$
}

Received: 27 June 2017/Accepted: 12 August 2017/Published online: 20 August 2017

(c) The Author(s) 2017. This article is an open access publication

\begin{abstract}
The versatility of superparamagnetic iron-oxide nanoparticles (SPIONs) have been extensively investigated, especially for their applications in therapeutics and diagnostics. Considering their intriguing feature of contrasting agent, in terms of medical applications, it is still in its infancy. Various physicochemical parameters like magnetism, crystallinity, and optical parameters contribute to their better contrasting agent. In this study, SPIONs were synthesized with different concentrations of precursor iron molecular solution in the presence of magnetic field and the optimum concentration of precursor iron molecular solution was determined as $0.133 \%$. SPIONs obtained at optimum concentration were further analyzed by both microscopic and spectroscopic analysis. The difference occurred in the elemental nature of SPIONs as they were synthesized in the magnetic field out of precursor iron molecular solution was analyzed with a specific reference to NMR spectroscopy. SPIONs as contrasting agent against $\mathrm{X}$-ray imaging was also investigated in quail's egg.
\end{abstract}

Keywords SPIONs · NMR spectroscopy $\cdot$ XRD · Contrasting agent

Antony V. Samrot

antonysamrot@gmail.com

1 Department of Biotechnology, Sathyabama University, Jeppiaar Nagar, Rajiv Gandhi Salai, Chennai, Tamil Nadu 600119, India

\section{Introduction}

Over the decades, various methods of synthesizing the SPIONs are investigated and reported which includes chemical and physical methods like thermal decomposition, microwave-assisted synthesis, etc. (Kandasamy and Maity 2015; Jadhav and Bongiovanni 2012). While the iron-oxide nanoparticles are synthesized by chemical method, there are a number of factors involved, especially the morphology of the iron-oxide particle depends on the processes like nucleation, growth, aggregation, and adsorption of impurities (Mohapatra and Anand 2010). Moreover, during chemical synthesis of SPIONs, unpaired electrons' spins in radicals experience various interactions (e.g., spin-orbit, exchange, hyperfine, and Zeeman) and, as a result, carry out coherent and stochastic motions (Schulten and Weller 1978). The only metal nanoparticles approved by Food and Drug Administration are iron-oxide nanoparticles (Colombo et al. 2012). Among the various nanoparticles, superparamagnetic nanoparticles (SPIONs) particularly magnetite $\left(\mathrm{Fe}_{3} \mathrm{O}_{4}\right)$ and maghemite $\left(\gamma-\mathrm{Fe}_{2} \mathrm{O}_{3}\right)$, are researched for their resourcefulness in medical applications like theranostics, hyperthermia, drug delivery, magnet influenced radionuclide therapy, contrasting agents for imaging purposes, and MRI (Schleich et al. 2013; Bolden et al. 2008; Kievit and Zhang 2011; Cortajarena et al. 2014; Jalilian et al. 2009; Talelli et al. 2009: Shubayev et al. 2009; Lee and Hyeon 2012). SPIONs can be used for targeted drug delivery as it can be guided through external magnets as they possess superparamagnetism, and in addition, they do not show magnetic interaction once the external magnetic field is stopped ( $\mathrm{Li}$ et al. 2016; Leder et al. 2015). Nanoparticles have found their application in imaging, where they are used as contrasting agents. Gold and iodine-based compounds are commonly used in 
computed tomography (CT) contrasting agents (Thomas et al. 2013; Wang et al. 2011; Giljohann et al. 2010; Hasebroock and Serkova 2009). These metal nanoparticles can scatter and/or absorb the high energy gamma/X-ray radiations; thus, imaging/targeting tumor tissues at deeper location using X-rays is possible (Kwatra et al. 2013), but they have their limitations which depend on the stability inside biological system as well as the chemical nature of particles (Generalov et al. 2015; Retif et al. 2015; Nunes et al. 2006). This therapeutic approach solves the X-ray penetration problem and allows treatment of deep tumors using lower radiation dose than the conventional radiotherapy (Retif et al. 2015). Spatial Frequency Heterodyne Imaging (SFHI), an X-ray imaging technique, has been utilized SPIONs as contrast agents as well as low dose radiation therapy (Rand et al. 2011, 2015; Klein et al. 2014). For the imaging purposes, mixed phase of $\gamma-\mathrm{Fe}_{2} \mathrm{O}_{3}$ and $\mathrm{Fe}_{3} \mathrm{O}_{4}$ of size between 9 and $20 \mathrm{~nm}$ is commonly utilized (Klein et al. 2014). Having in mind the ample potentials of SPIONs, SPIONs were synthesized by chemical co-precipitation method and a preliminarily attempt was made to investigate the effect of SPIONs as contrasting agent in visualizing Quail's egg.

\section{Materials and methods}

\section{Materials used}

In this research, analytical grade chemicals were used throughout the experiment. For the synthesis of iron-oxide nanoparticles, ferrous sulphate heptahydrate, extra pure $\left(\mathrm{FeSO}_{4} \cdot 7 \mathrm{H}_{2} \mathrm{O}\right)$ was purchased from HiMedia Laboratories, Mumbai, India. Ferric chloride hexahydrate $\left(\mathrm{FeCl}_{3} \cdot 6 \mathrm{H}_{2} \mathrm{O}\right)$ was purchased from Thomas Baker (Chemicals) Pvt. Ltd., Mumbai, India. Tetramethyl ammonium hydroxide $\left(\mathrm{C}_{4} \mathrm{H}_{13} \mathrm{NO}\right)$, formaldehyde $\left(\mathrm{CH}_{2} \mathrm{O}\right)(41 \%)$, and acetone $\left(\mathrm{C}_{3} \mathrm{H}_{6} \mathrm{O}\right)$ were bought from SD Fine-Chem Limited, Mumbai, India. Magnets with the strength of 30 Gauss were used for creating magnetic field. Bar magnets with the strength of 50 Gauss were used for magnetic separation of the particles during washing processes. For TEM imaging, Formvar/Carbon copper grids \# 300 mesh size were purchased from Toshniwal Brothers (SR) Private Limited. For the application of X-ray, the digital X-ray facility of Cratis Hospital, Bangalore, Karnadaga, India was used. For the synthesis of SPIONs, nitrogenized double distilled water was used.

\section{Synthesis of SPIONs}

$0.1 \mathrm{~g}$ of $\mathrm{FeCl}_{3} 6 \mathrm{H}_{2} \mathrm{O}$ and $\mathrm{FeSO}_{4} 7 \mathrm{H}_{2} \mathrm{O}$ were dissolved separately in $1 \mathrm{ml}$ of nitrogenized double distilled water. Both the iron sources were mixed together. $500 \mu$ of the above solution was added to $500 \mu \mathrm{l}$ of tetramethyl ammonium hydroxide and vortexed for 5 mins. Different concentrations of the obtained black precipitates were washed with acetone [black precipitate $(\mu \mathrm{l})$ : acetone $(\mathrm{ml})-0.03: 1$, 0.025:1, 0.02:1, 0.015:1, 0.01:1, and 0.005:1]. Magnetic field was applied and acetone was slowly pipetted out. Each derived pellet was dried in the hot air oven at $60{ }^{\circ} \mathrm{C}$ for half an hour. The obtained pellet $(0.003,0.0025,0.002$, $0.0015,0.001$, and 0.0005 ) was dissolved in $700 \mu \mathrm{l}$ of Formaldehyde. Then, $300 \mu \mathrm{l}$ of nitrogenized double distilled water was added dropwise with simultaneous vortexing. Again, $500 \mu \mathrm{l}$ of formaldehyde solution was added dropwise with simultaneous vortex. The percentage solution obtained was as follows- $0.2,0.166,0.133,0.1,0.066$, and $0.033 \%$. Different concentration of solution was prepared to find the optimal condition of production of monodispersed nanoparticles. Thus, precursor iron molecular solution was prepared. This precursor iron molecular solution was kept in magnetic field for 2 days for the synthesis of SPIONs (Fig. 1). After 2 days of incubation, the obtained SPIONs were dispersed into the same solution. The optimum concentration of precursor iron molecular solution for the synthesis of SPIONs was determined by analyzing the $\mathrm{pH}$ and calorimetric reading at $720 \mathrm{~nm}$.

\section{Microscopy analysis}

\section{Transmission electron microscopy}

TEM images were taken in TEECNAI G2 Spirit Biotwin$120 \mathrm{kV}$. Prior to the imaging, the samples were loaded in

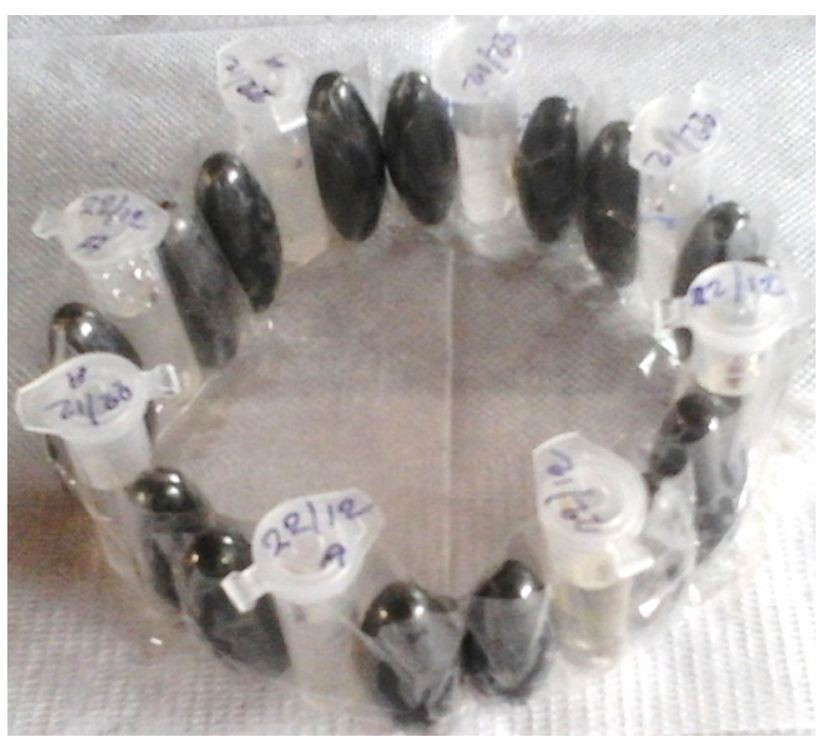

Fig. 1 Precursor ironic molecular solution kept in the magnetic field for the synthesis of SPIONs 
Formvar/Carbon copper grids \# 300 mesh. TEM images were taken for the SPIONs synthesized at all the six molecular concentrations. Morphologically satisfactory particles synthesized in the optimum concentration were subjected for the other microscopic and spectroscopic and $\mathrm{X}$-ray studies.

\section{Scanning electron microscopy}

SEM images of synthesized SPIONs were taken in Zeiss Ultra55. Prior to the imaging, the particles were dried and were done with gold coating in Quorum, Q 150R ES.

\section{Atomic force microscopy}

Samples were transferred onto a clean glass coverslip. AFM images were taken in Bruker, Dimension icon model.

\section{Spectroscopy analysis}

$U V-V i s$ spectroscopy

UV-Vis absorbance value was obtained between 200 and $800 \mathrm{~nm}$ using UV 3600 Shimadzu UV-Vis spectrophotometer.

\section{Fourier transform infrared spectroscopy}

FTIR spectra were recorded using IR Affinity 1S (Shimadzu, Japan). The sample for the spectra was mounted over a clean glass slide and the reading was recorded with transmission mode scan in the spectral region of $4000-500 \mathrm{~cm}^{-1}$.

\section{Raman spectroscopy}

Raman spectra were taken in LabRam HR 800 model of Horiba Jobinyvon. The sample was dried in a clean glass slide and $785 \mathrm{~nm}$ diode laser was used for deriving the spectrum.

\section{$X$-ray diffraction spectroscopy}

XRD (FXRD) spectra were taken in Smart lab XRD model of Rigaku, and Copper $\mathrm{K} \alpha$ was used as the X-ray source and the power used was $1.2 \mathrm{kw}$.

\section{X-ray photoelectron spectroscopy (XPS)}

XPS analysis was done in Ultra DLD model of Kratos. Monochromatic source $\mathrm{Al} \mathrm{K} \alpha$ at the condition of $10 \mathrm{~K} \mathrm{eV}$ as accelerating voltage and $9 \mathrm{~mA}$ current $(90 \mathrm{~W})$ at the vacuum of $2 \mathrm{e}^{-9}$ torr was used. In addition to Fe, spectrum for metal oxide, carbon, and nitrogen was also taken to understand the interstitial elements in the SPIONs.

\section{NMR spectroscopy}

NMR analysis was done in Bruker NMR Spectrometer, using BBFO Probe. The analysis was done before the application of the magnetic field (precursor iron molecular solution) and after the application of the magnetic field (after the synthesis of SPIONs). It was done for the hydrogen and carbon.

\section{Zeta potential analysis}

To understand the stability of the SPIONs, zeta potential value was recorded in zeta potential analyzer using Brookhaven ZetaPALS.

\section{Superconducting quantum interference device (SQUID)}

SQUID from quantum design, the material properties and measurement system (MPMS), was used to take the magnetic hysteresis and also the magnetic measurements at both (field cooled) FC and (zero-field cooled) ZFC modes.

\section{X-ray imaging of quail's egg}

Quail's eggs were used for observing the contrasting effect of the SPIONs. $2 \mathrm{ml}$ of egg substance was removed with the help of a syringe. $1 / 1.8 \mathrm{ml}$ of SPIONs in distilled water was injected into the egg. It was then done with gentle hand shaking for a minute to get the particles dispersed inside the egg. It was then subjected for hospital-based X-ray imaging. For observing the contrasting effect, Wipro G machine with the capacity of 300 milli ampere (ma) was used. For taking the X-ray film, the computerized radiography (CR) of Fujifilm FCR Prima $\mathrm{T}$ was used. To observe whether the SPIONS are having magnetic property inside the system, a one bar magnet was kept near the SPIONs' dispersed egg and the X-ray image was taken. While taking the images, the eggs were kept over a cotton flat to prevent them from rolling down the X-ray cassette.

\section{Results and discussion}

\section{Synthesis of SPIONS}

$\mathrm{pH}$ and calorimetric values of six different concentrations precursor ironic molecular solution and the resultant SPIONs were analyzed (Tables 1,2 ). The SPIONs formed at $0.133 \%$ concentration were found to be dispersed for 
Table 1 Colorimetric readings taken at $720 \mathrm{~nm}$ for the precursor ironic molecular solution (before the synthesis of SPIONs) and after the synthesis of SPIONs

\begin{tabular}{lllllr}
\hline Concentration & $0.2 \%$ & $0.166 \%$ & $0.133 \%$ & $0.1 \%$ & $0.066 \%$ \\
\hline Precursor iron molecular solution & 0.455 & 0.456 & 1.682 & 1.333 & 1.876 \\
After the synthesis of SPIONs & 1.840 & 1.861 & 1.660 & 2.000 & 2.000 \\
\hline
\end{tabular}

Table $2 \mathrm{pH}$ values taken for precursor iron molecular solution (before the synthesis of SPIONs and after the synthesis of SPIONs)

\begin{tabular}{llllllr}
\hline Concentration & $0.2 \%$ & $0.166 \%$ & $0.133 \%$ & $0.1 \%$ & $0.066 \%$ & $0.033 \%$ \\
\hline Precursor iron molecular solution & 8.32 & 8.16 & 7.90 & 8.05 & 8.71 & 7.82 \\
After the synthesis of SPIONs & 7.76 & 7.88 & 7.86 & 7.91 & 7.81 & 7.39
\end{tabular}

longer duration, even for a month. The increase in absorbance and change in $\mathrm{pH}$ in the further dilution was due to lack of monodispersion. It is already reported that the colloidal stability is greatly influenced by $\mathrm{pH}$ and which is contributed by organic acids (Tombácz et al. 2013, 2014). The reduction in $\mathrm{pH}$ value could be attributed by the disappearance of organic molecular hydrogen from the solution into the SPIONs as the interstitial element which is further substantiated with NMR results. Moreover, the fine saturation of precursor molecules in appropriate organic solvent was expected at this concentration and the type and strength of the magnetic field applied for better tuning of SPIONs could also be a better possibility.

\section{Microscopic results}

To determine the optimum working concentration for the further experiment, the morphology of the SPIONs synthesized in the six different concentrations was imaged under TEM (Fig. 2a-f). SPIONs synthesized at $0.133 \%$ of concentration were found to be morphologically finest among the other concentrations, and the size was found to be ranging between 15 and $20 \mathrm{~nm}$ (Fig. 2c). More uniform and less aggregated particles were observed at this specific concentration. In case of the increased concentration, more aggregation was found (Fig. 2a, b). In lesser concentrations, comparatively the morphology was not uniform (Fig. 2d, e) and a considerable volume of molecules remained not involved in the magnetic field-mediated reaction which was observed as a light black shade as it appears in the image (Fig. 2f). The optimal condition for the SPION formation was at $0.133 \%$ which involved various factors like reducing agents and concentration of precursor molecule used. For the present study, those SPIONs synthesized at $0.133 \%$ were further studied by SEM and AFM imaging. SEM and AFM analysis of the particles showed that the particles were well dispersed and size below $20 \mathrm{~nm}$ (Fig. 3a, b).

\section{Spectroscopic analysis}

UV-Vis

SPIONs synthesized at $0.133 \%$ of molecular concentration showed absorbance spectral ranges between 250 and $300 \mathrm{~nm}$ (Fig. 4). Among the respective peaks, the absorbance maximum at $250 \mathrm{~nm}$ is the characteristic peak for the SPIONs. This value is much closer to the already reported absorbance near $250 \mathrm{~nm}$ maxima for magnetic nanoparticles (Wang et al. 2014; Samrot et al. 2016). The other minor peaks observed may be due to the absorbance of the organic molecules.

\section{FTIR}

The FTIR spectrum of SPIONs manifests the prominent absorption bands located between 3384 and $2073 \mathrm{~cm}^{-1}$ which is considered the finger print region for iron-oxide nanoparticles (Fig. 5). Most of the bands are $\mathrm{O}-\mathrm{H}$ stretching/carboxylic acid $(\mathrm{m})$ and $-\mathrm{C} \equiv \mathrm{C}-$ which are characteristic of the interstitial organic molecules present among the crystalline phases of SPIONs. This is because of SPIONs synthesized in chemical method; the high absorption affinity was resulted from $\pi$-electron interactions with polar surface of magnetic nanoparticles, as well as complex bond formation at $\equiv \mathrm{Fe}-\mathrm{OH}$ sites (Tombácz et al. 2013).

\section{Raman spectroscopy}

According to the given Raman spectra (Fig. 6), the peaks at 220, 280, 400, and $470 \mathrm{~cm}^{-1}$ correspond to Eg mode and the peak at $600 \mathrm{~cm}^{-1}$ corresponds to $\mathrm{A} 1 \mathrm{~g}$ mode of the iron oxide. Similar peak ranges were already reported by Mitchell et al. (2015), which is arising when iron-oxide nanoparticles were subjected to $514.5 \mathrm{~nm}$ wavelength laser excitation. 
Fig. 2 TEM images of SPIONs synthesized in the magnetic field-mediated method at six different precursor iron molecular concentrations. a $0.2 \%$, b $0.166 \%$, c $0.133 \%$, d $0.100 \%$, e $0.066 \%$, and f $0.033 \%$

Fig. 3 Analysis of SPIONS under various microscopes. a SEM image of the SPIONs synthesized in the magnetic field with $0.133 \%$ precursor iron molecular solution. b AFM image of SPIONs synthesized in the magnetic field with $0.133 \%$ precursor iron molecular solution
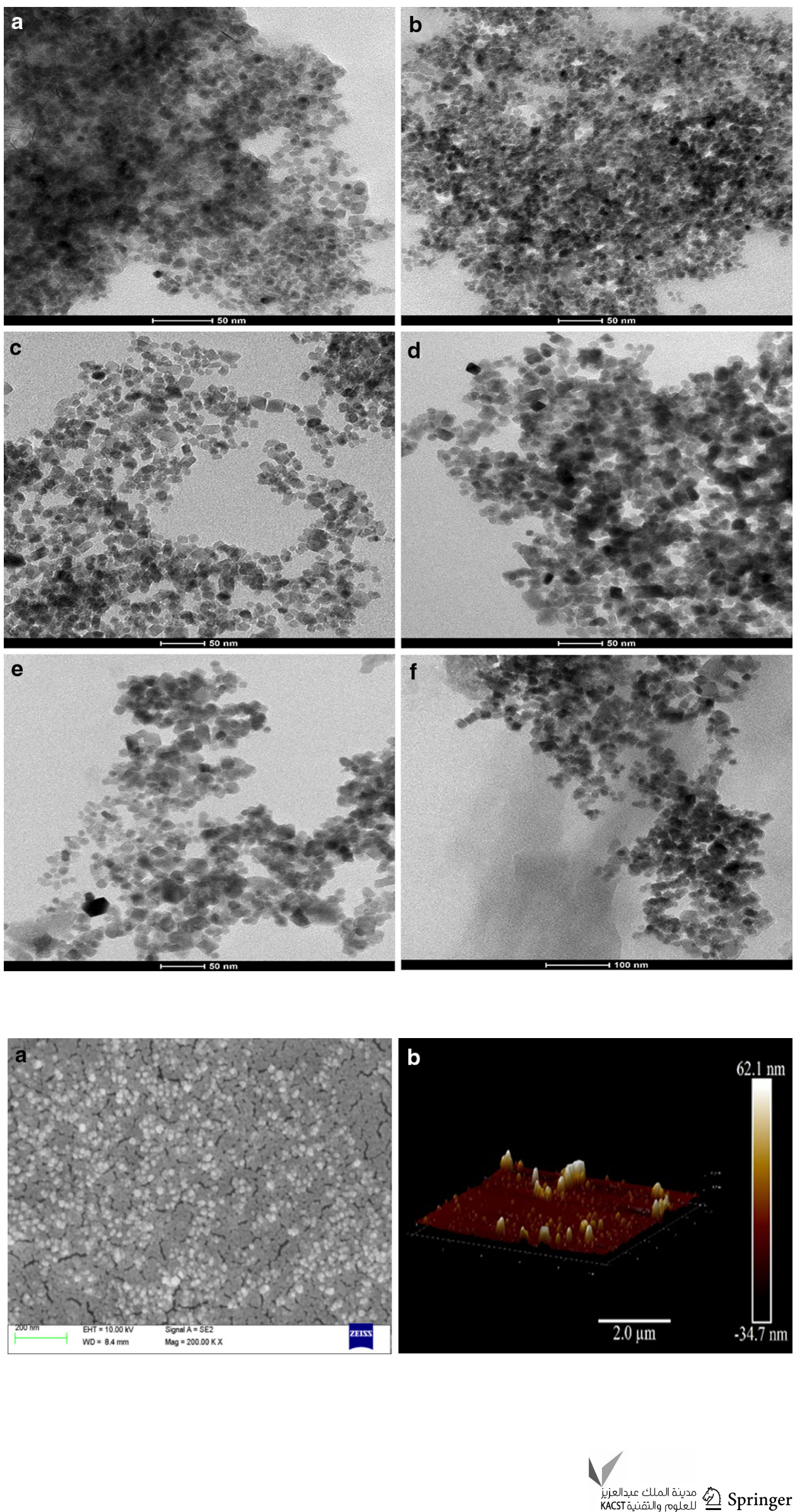


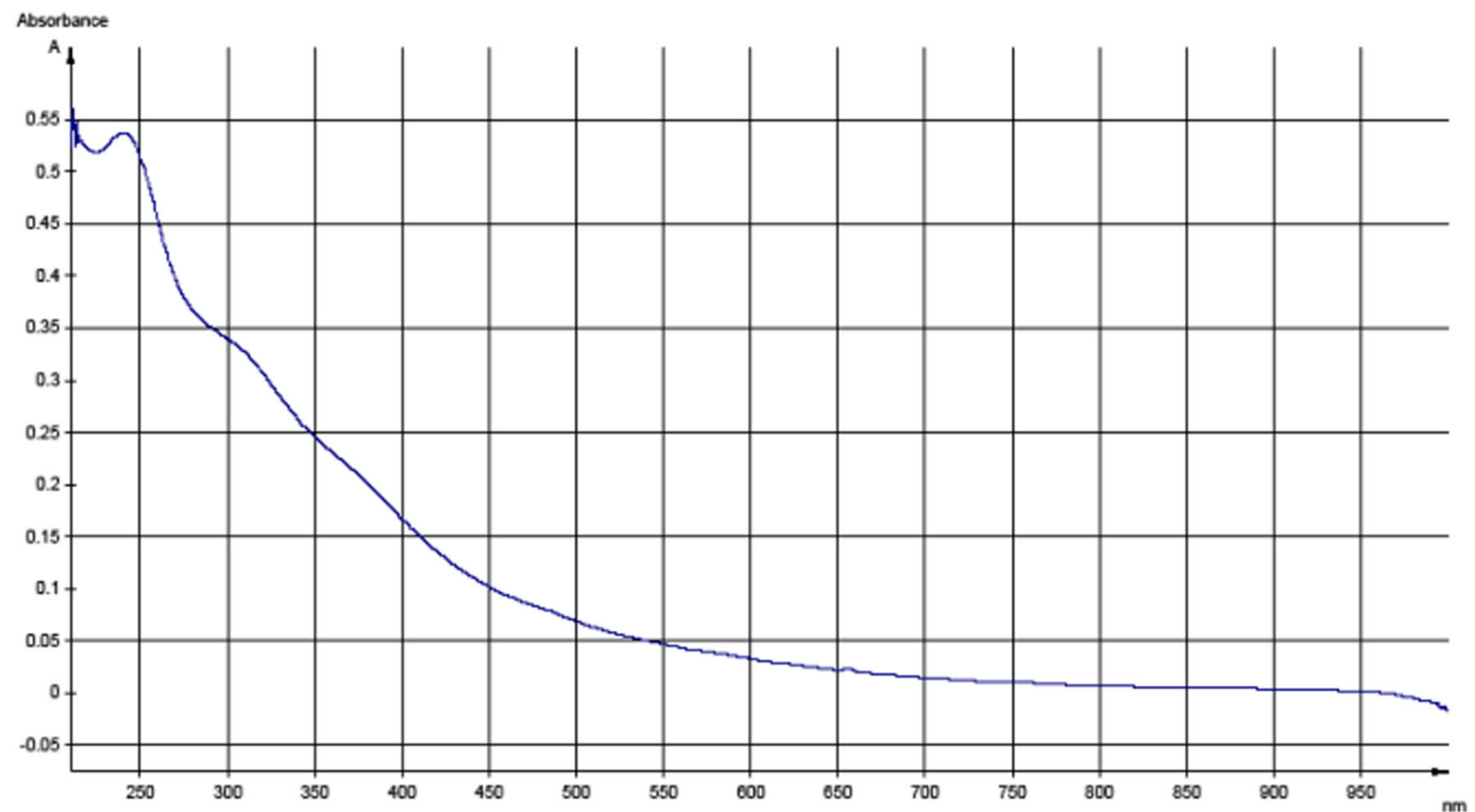

Fig. 4 UV-Vis spectra of SPIONs

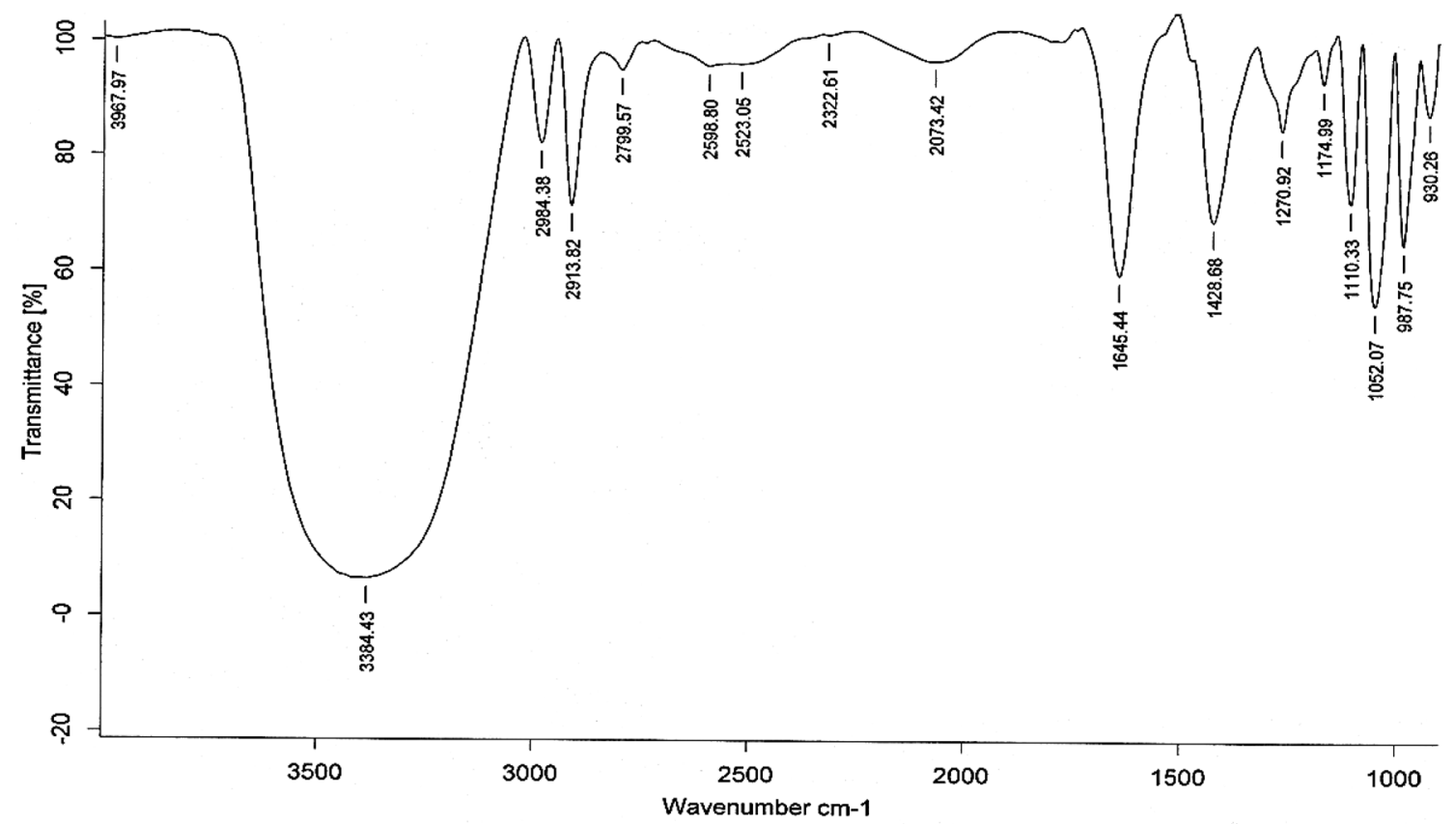

Fig. 5 FTIR spectra of the SPIONs

$X R D$

The X-ray diffraction analysis showed that the obtained SPIONs are crystalline in nature (Fig. 7). All the XRD peaks could be identified with $\gamma-\mathrm{Fe}_{2} \mathrm{O}_{3}$ structure in comparison with what is already reported by Layek et al. (2010).

\section{$X$-ray photoelectron spectroscopy}

XPS data support that the synthesized particle as $\mathrm{Fe} 2 \mathrm{p}_{3 / 2}$ metallic nature with mixed elements. As per the spectra (Fig. 8a), the binding energy for $\mathrm{Fe}_{2} \mathrm{O}_{3}$ occurs at $710.8 \mathrm{eV}$ and $\mathrm{FeO}$ at $709.6 \mathrm{eV}$, and $\mathrm{Fe}$ at $706.7 \mathrm{eV}$. A considerable amount of metal chloride was present in the SPIONs for 


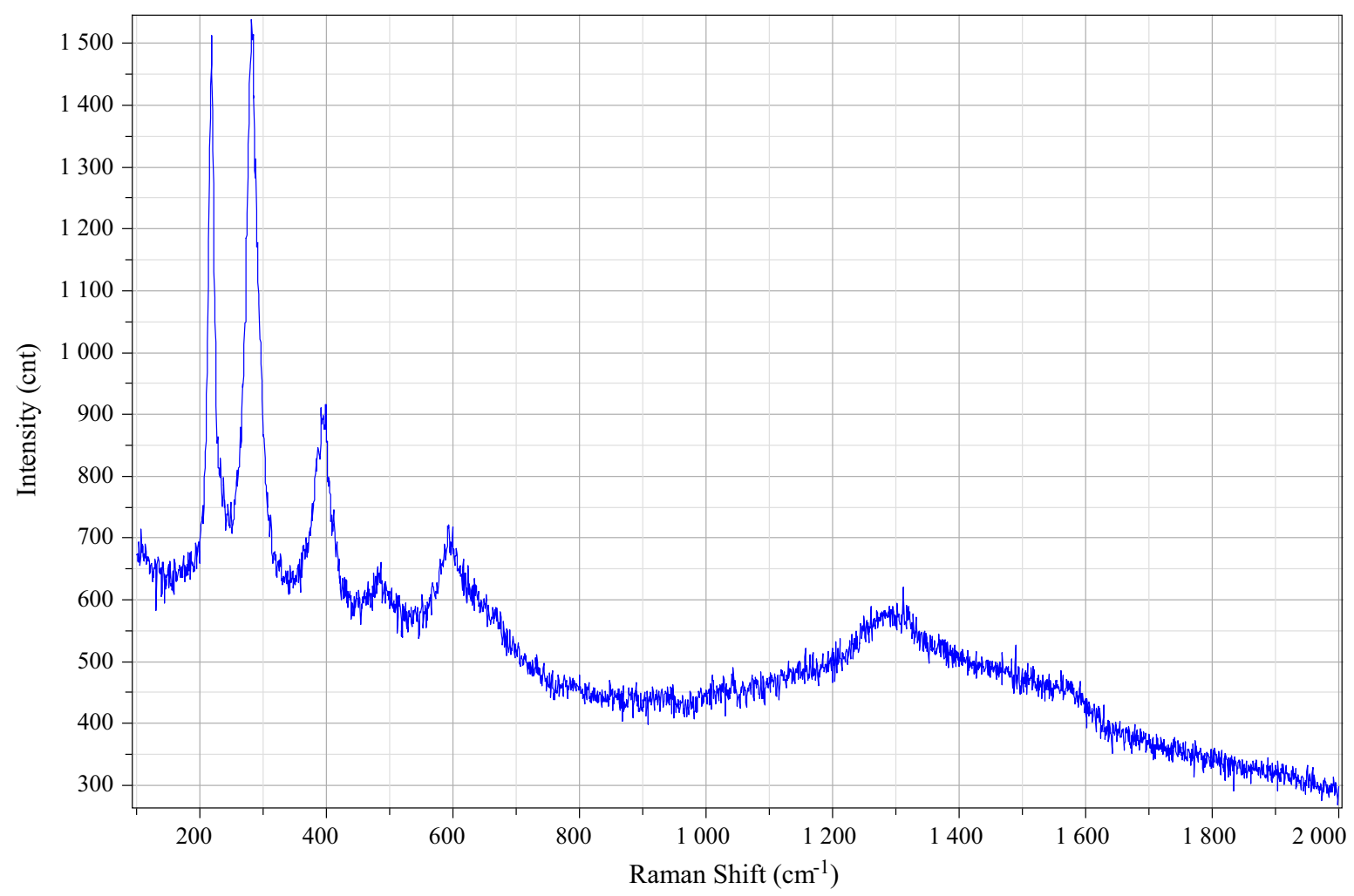

Fig. 6 Raman spectra of the SPIONs

Fig. 7 XRD spectra of SPIONs

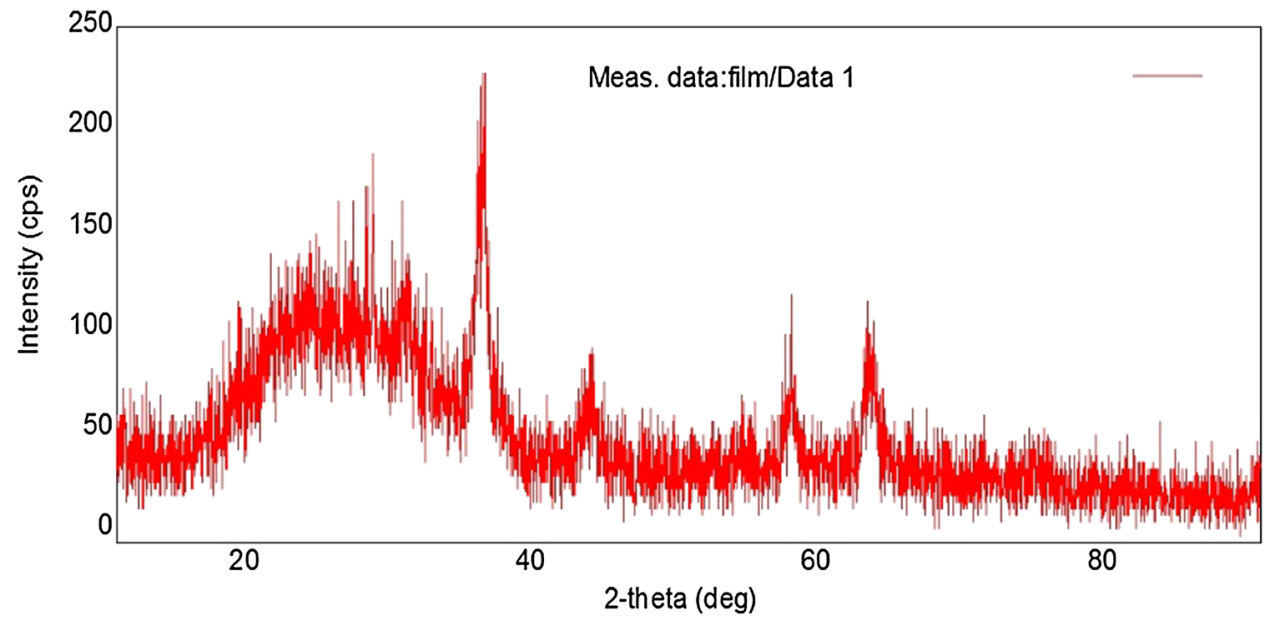

which the binding energy occurs at $710.4 \mathrm{eV}$ in the spectrum. The binding energy for oxygen was observed (Fig. 8b). Peak occurred in between 529 and $530 \mathrm{eV}$ was suggestive of metal oxide. Other than the metal oxide, minor level of organic oxygen was also detected. The binding energy observed at 531.5-532 eV was suggestive of $\mathrm{C}-\mathrm{O}$, and $\sim 533 \mathrm{eV}$ was suggestive of $\mathrm{C}=\mathrm{O}$ and $\sim 535$ was suggestive of $\mathrm{O}-\mathrm{F}_{X}$. The reason for the presence of organic oxygen is that since iron is a transition metal, which are capable of entrapping smaller atoms such as $\mathrm{H}$, $\mathrm{C}$, and $\mathrm{N}$ in their interstitial sites of their crystal lattices. These trapped atoms get bonded to the atoms of the transition elements (Yadav 2003). Moreover, significant presence of molecular carbon is detected in the SPIONs (Fig. 8c). The binding energy occurs at 284.8, 286, and $288.5 \mathrm{eV}$ was suggestive of $\mathrm{C}-\mathrm{C}, \mathrm{C}-\mathrm{O}-\mathrm{C}$, and $\mathrm{O}-\mathrm{C}=\mathrm{O}$, respectively. Though it is likely to occur the adventitious carbon contamination during the chemical process, the 

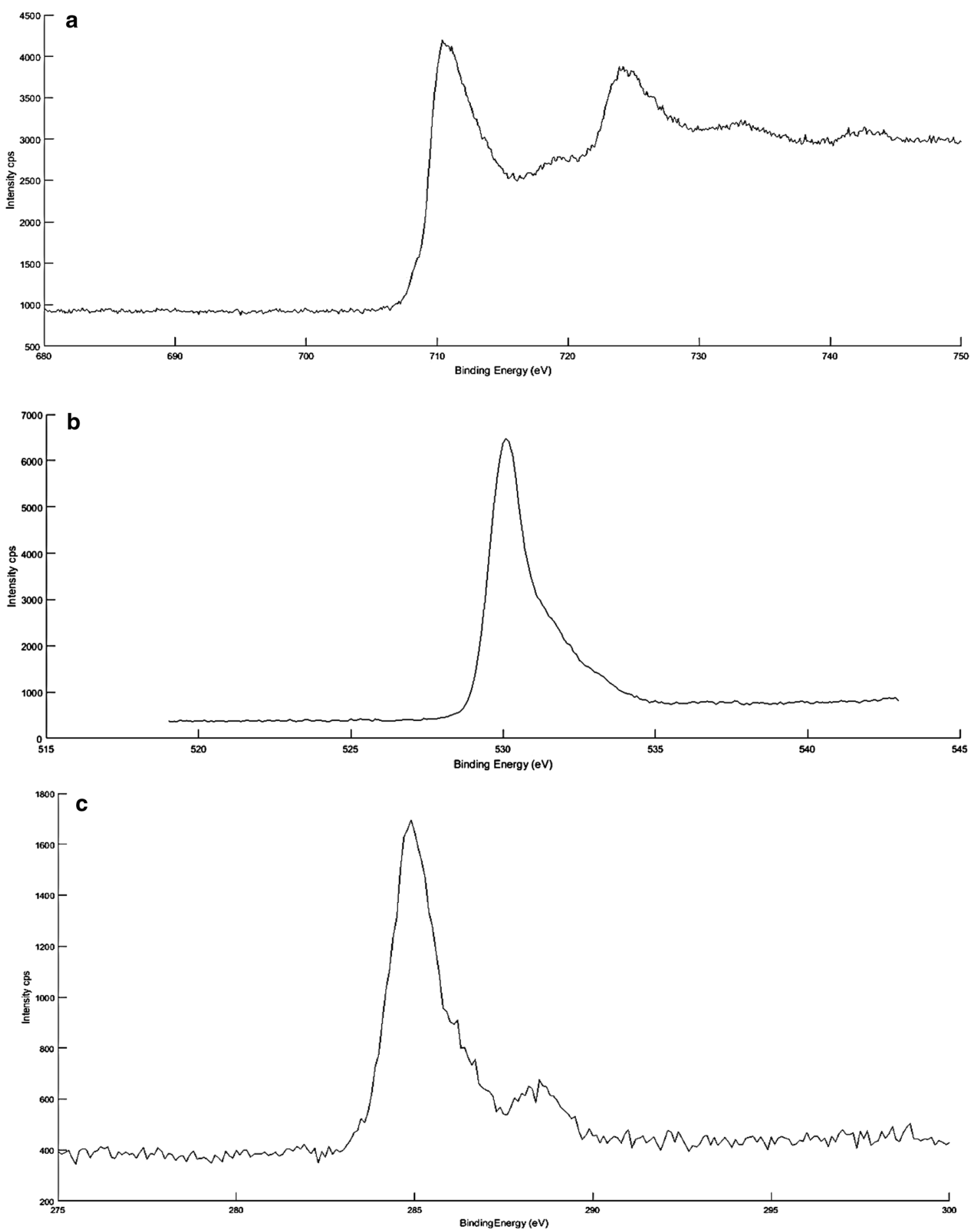

Fig. 8 XPS spectra of SPIONs: a Fe2p3/2; b for metal oxide; $\mathbf{c}$ for adventitious carbon

magnetic field-mediated synthesis would also be another reason for this phenomenon. The occurrence of interstitial elements like hydrogen and carbon in magnetic field-mediated synthesis is further substantiated in a preliminarily scale by NMR spectra which was done for carbon and hydrogen of precursor ironic molecular solution and then the SPIONs synthesized in the magnetic field.
$N M R$

NMR was used to study the difference occurred before and after the synthesis of SPIONs. Special focus was given to hydrogen and carbon. The presence of hydrogen (Fig. 9a, b) and the presence of carbon (Fig. 9c, d) were examined in both precursor ironic molecular solution and the synthesized 

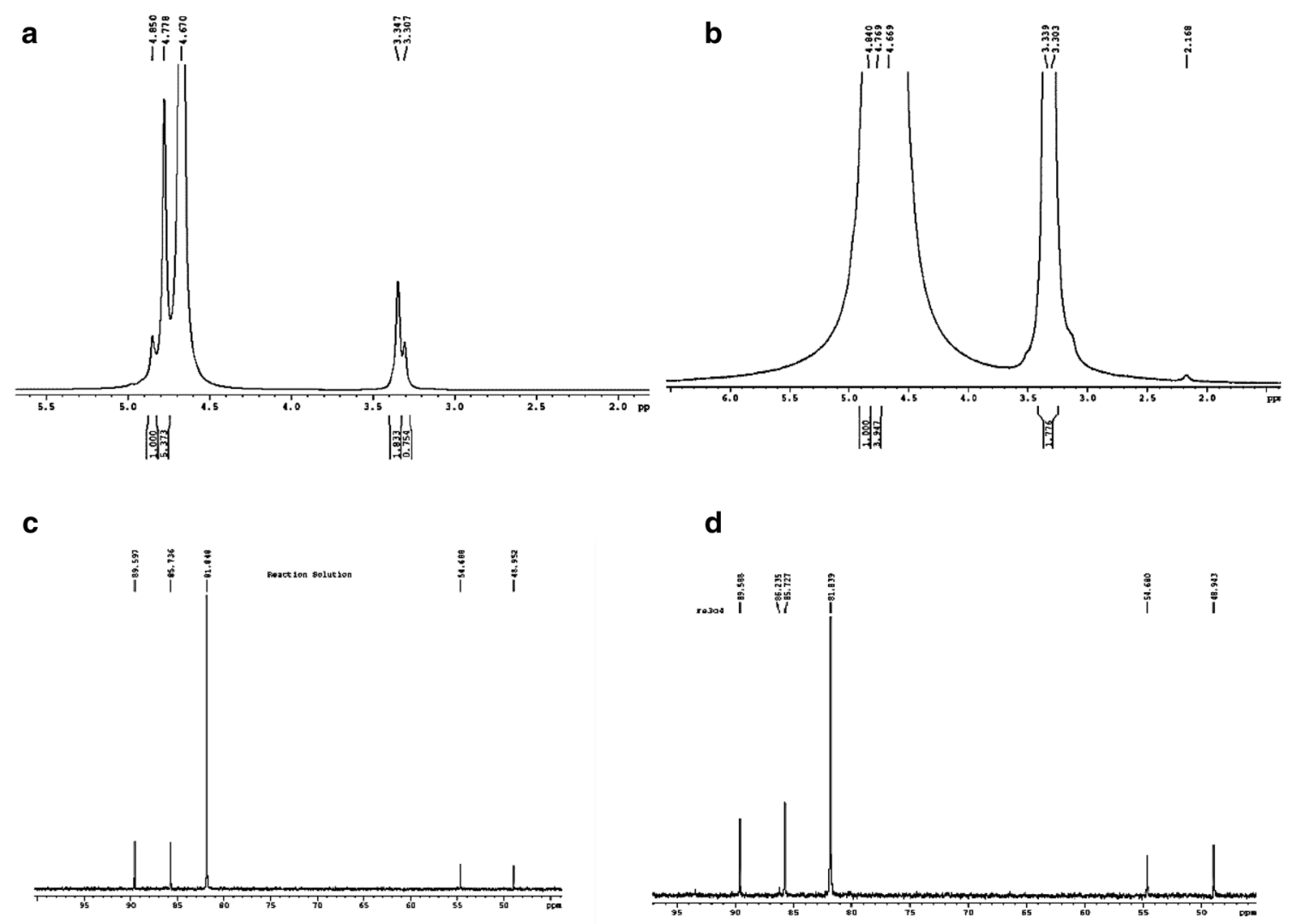

Fig. 9 a NMR spectra for hydrogen of precursor iron molecular solution, i.e., before the synthesis of SPIONs in the magnetic field; b NMR spectra for hydrogen for SPIONs synthesized in the magnetic

field; c NMR spectra of carbon of precursor iron molecular solution; d NMR spectra of carbon of SPIONs synthesized in the magnetic field

Table 3 NMR spectral values

\begin{tabular}{|c|c|c|c|c|c|c|c|}
\hline Peak no. & Sample & 1 & 2 & 3 & 4 & 5 & 6 \\
\hline \multirow[t]{2}{*}{ Hydrogen } & (a) & Nil & 3.307 & $3 . \underline{447}$ & 4.670 & $4.7 \underline{78}$ & $4.8 \underline{50}$ \\
\hline & (b) & 2.168 & $3.30 \underline{3}$ & 3.339 & $4.6 \underline{69}$ & $4.7 \underline{69}$ & $4.8 \underline{40}$ \\
\hline \multirow[t]{2}{*}{ Carbon } & (a) & 48.952 & 54.688 & 81.848 & 85.736 & Nil & $89.5 \underline{97}$ \\
\hline & (b) & 48.943 & 54.680 & 81.839 & 85.727 & 86.235 & $89.5 \underline{88}$ \\
\hline
\end{tabular}

(a) Spectral values of hydrogen obtained for precursor iron molecular solution; (b) spectral values of hydrogen obtained for SPIONs; (c) spectral values of carbon obtained for the precursor iron molecular solution; (d) spectral values of carbon obtained for SPIONs

The difference in spectral values in between precursor iron molecular solution and SPIONs is underlined. In addition, the emergence of new spectra is given in bold

SPIONs. The difference in the resonance values for hydrogen and carbon was taken for precursor ironic molecular solution and the synthesized SPIONs (Table 3). The difference in the spectral values could be equated with change in the $\mathrm{pH}$ values before and after the synthesis of SPIONs. In the spectrum, it could be noted that there is an emergence of two peaks at 2.168 and 86.235 for hydrogen and carbon, respectively, in precursor iron solution, whereas it was considerably decreasing in the spectral values after the synthesis of SPIONs; this could be interpreted as the phenomenon of molecular convergence occurred in the magnetic field-mediated synthesis of SPIONs.

\section{Zeta potential}

The zeta potential value $(\mathrm{mV})$ is derived at -14.5 (Fig. 10). The importance of zeta potential is already reported in detail (Salopek et al. 1992). Moreover, the particles are negatively charged and the conductivity is measured at $(\mathrm{mS} / \mathrm{cm}) 1.28$. 
Fig. 10 Zeta potential analysis of the SPIONs

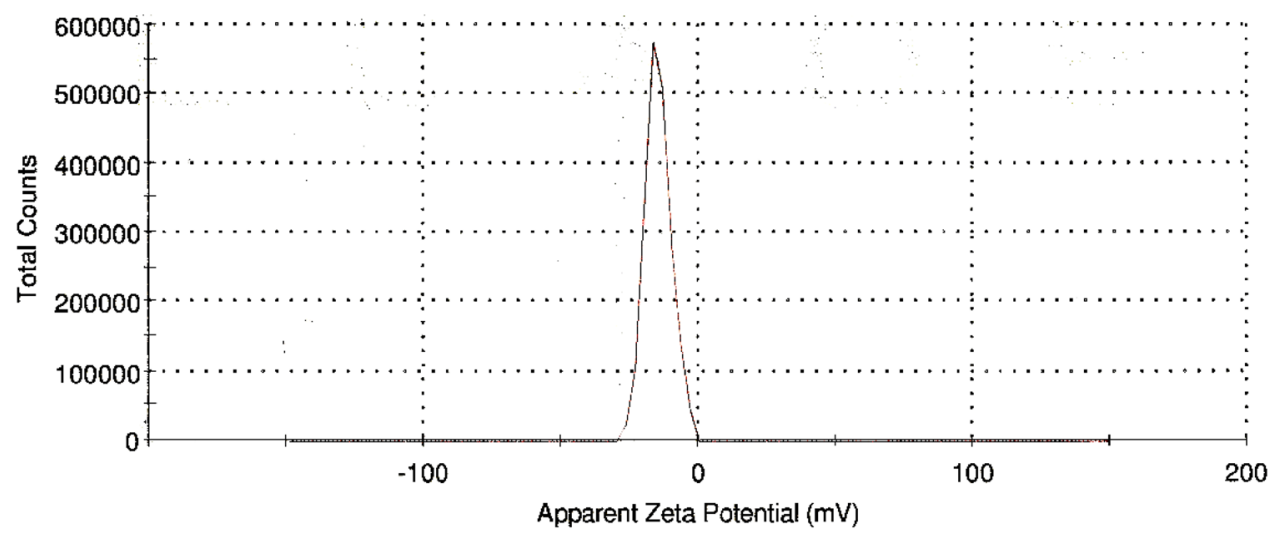

\section{SQUID}

The shape of the hysteresis curve for the particles was normal and tight with no hysteresis losses as expected for superparamagnetic iron-oxide nanoparticles (Fig. 11a). Only for temperatures below, $T_{\mathrm{B}}$ does the moment stay fixed in one direction during the time of measurement. Under low applied field, a high magnetization $\left(M_{\mathrm{S}}\right)$ and the coercivity $\left(H_{\mathrm{C}}\right)$ of the SPIONs are about $0.002 \mathrm{emu}$ (DC moment). Magnetization values were found to be below the $0.5 M_{\mathrm{S}}$ for $40 \mathrm{~nm}$ cubic particles (MartinezBoubeta et al. 2013). In our study, the $M_{\mathrm{S}}$ was lesser for our particle, as smaller particles have smaller magnetization (Martinez-Boubeta et al. 2013; Disch et al. 2012). To test the magnetic susceptibility, the temperature was held constant at $300 \mathrm{~K}$ for hysteresis measurement at the applied magnetic field 5T. Temperature-dependent magnetization (FC and ZFC) of the SPIONs was studied. In case of FC magnetization measurement, the sample was cooled in the presence of magnetic field to a temperature of $5 \mathrm{~K}$ to the magnetization which was recorded up to $400 \mathrm{~K}$ (Fig. 11b). Accordingly, there are different transition fluctuations observed. There was a steady increase in the magnetic moment as the temperature increased up to $200 \mathrm{~K}$ and a sudden decrease at $300 \mathrm{~K}$, and again an immediate increase up to $300-350 \mathrm{~K}$, and finally a sudden fall at $400 \mathrm{~K}$. The reduction in coercivity was observed mean particle size that was decreased (Martinez-Boubeta et al. 2013). Transition fluctuation in magnetic moment probably due to the different molecular arrangements occurred when the particles were formed in the magnetic field. In case of zero-field cooled (ZFC) mode, the sample was initially lowered to $5 \mathrm{~K}$ without any magnetic field applied. At the lowest temperature, a very week magnetic field was applied, and then, the magnetization was recorded as the temperature increased up to $400 \mathrm{~K}$ (Fig. 11c). At ZFC moments, the initial value is relatively very small; which means that the magnetic moments are frozen at this temperature $(5 \mathrm{~K})$. The magnetic moment
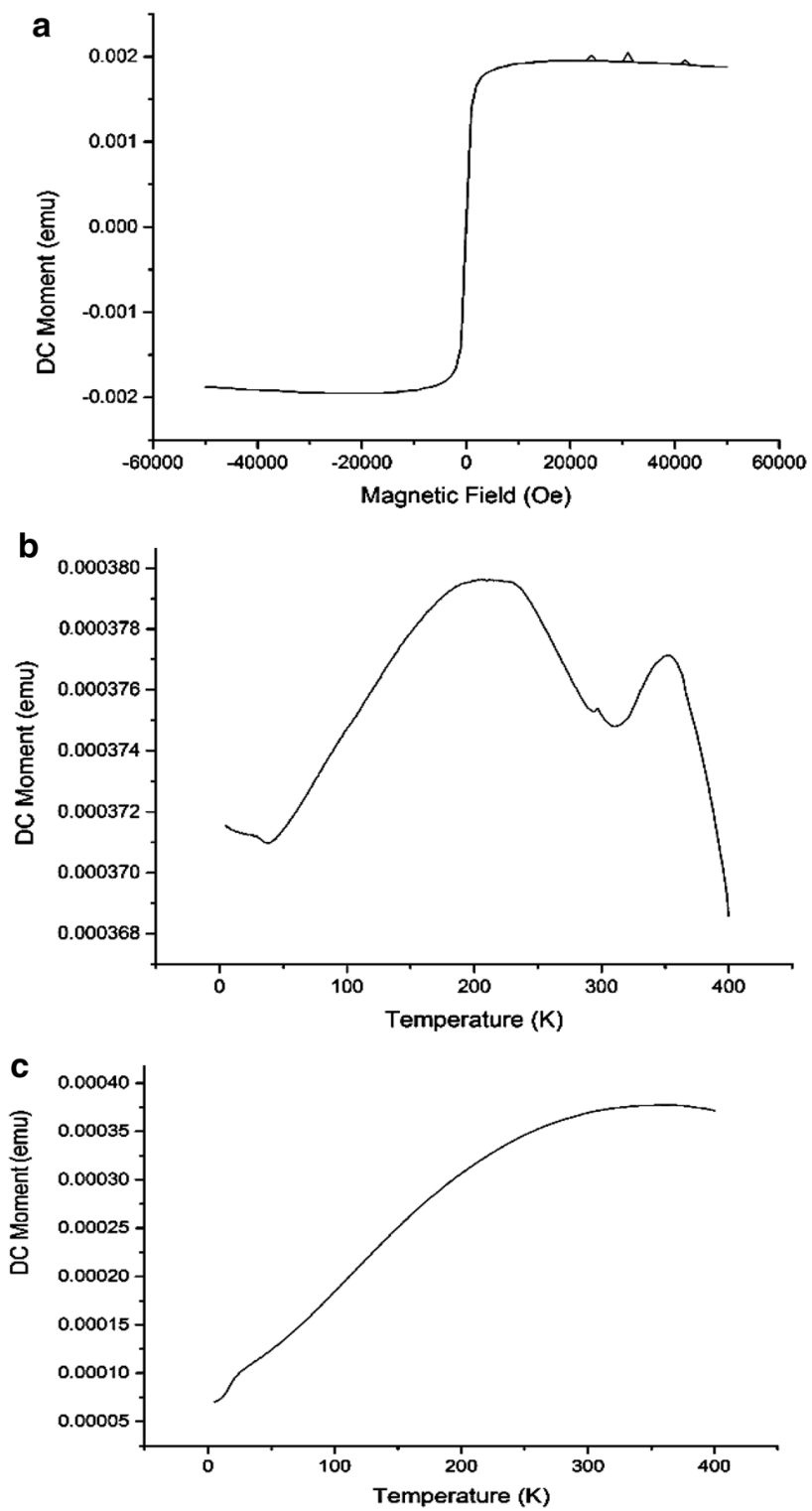

Fig. 11 SQUID magnetic analysis. a Hysteresis of the SPIONs synthesized in the magnetic field-mediated method at $0.133 \%$ of precursor iron molecular solution; $\mathbf{b}$ field cooled (FC) magnetization measurement; c zero-field cooled (ZFC) magnetization measurement 
Fig. 12 X-ray radio sensitizing effect of SPIONs in quail's egg. a X-ray image of control egg; b X-ray image of control egg with under applied magnetic field; c X-ray image of experimental egg with SPIONs dispersed in it; $\mathbf{d} \mathrm{X}$-ray radio sensitizing effect of SPIONs dispersed in the egg under applied magnetic field
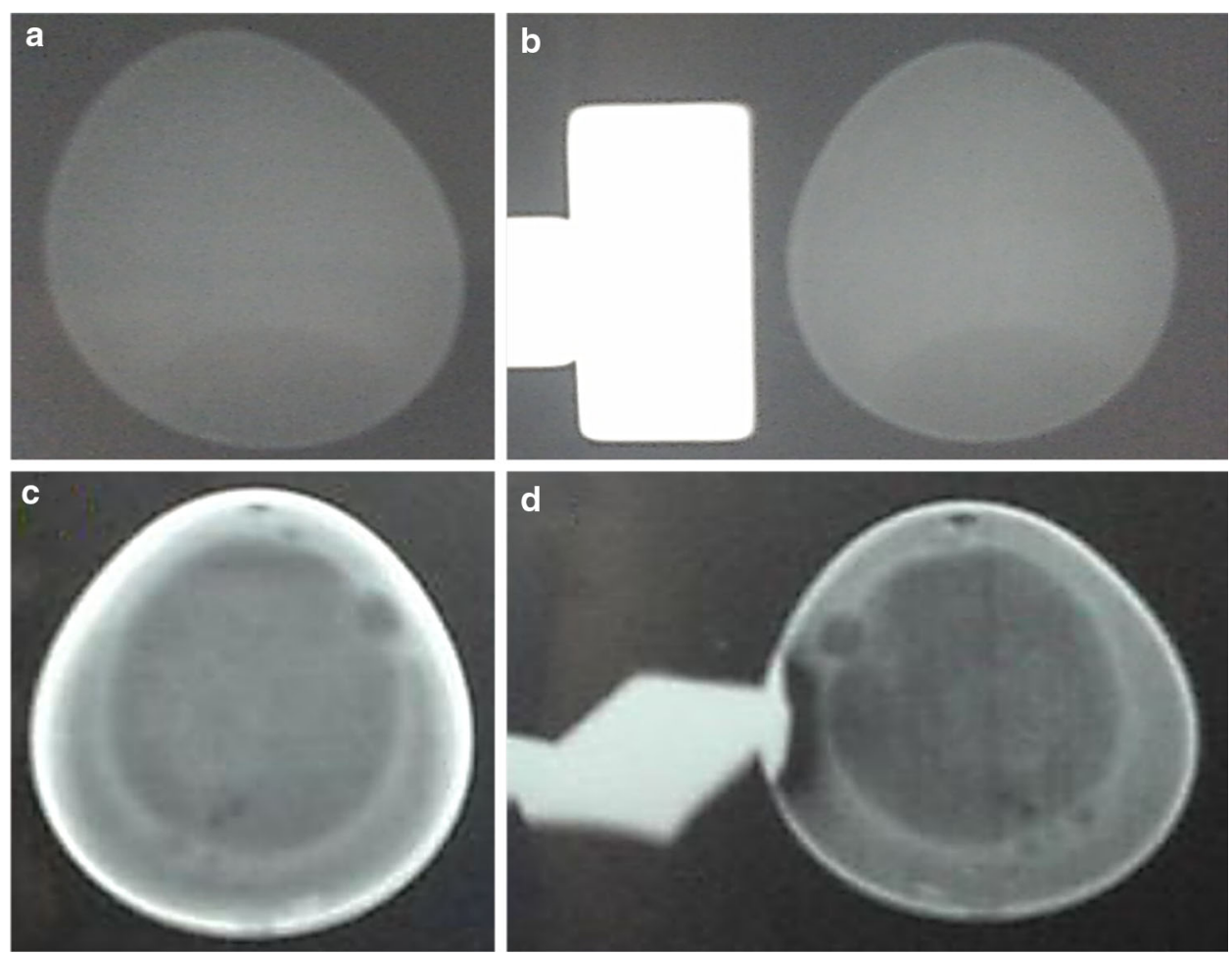

increased as the temperature was increased and reached a maximum at the blocking temperature (about $350 \mathrm{~K}$ ). Both for FC and ZFC moments, a common saturation magnetization was found at $350 \mathrm{~K}$ which is considered here as the $T_{B}$ for SPIONs synthesized in the magnetic field-mediated method. SPIONs synthesized in this study were found to withstand to temperature up to $350 \mathrm{k}$, and thus, it finds application in hyperthermia where temperature rises $315 \mathrm{~K}$; at this temperature, it initiates apoptosisinduced cell death (Fuller et al. 1994; Harmon et al. 1991).

\section{$\mathrm{X}$-ray imaging of quail's egg}

SPIONs were helping in visualizing the quail's egg clearly and they were enhancing the effect of X-ray and it could be used as contrasting agent in X-ray imaging. Even the magnetite was found to obey the external applied magnetic field (Fig. 12c, d). The commonly used computed tomography (CT) contrast agents are gold and iodine-based compounds; the contrasting agents block X-ray and enhance the image quality but these contrasting agents with side effects or cost effective (Thomas et al. 2013; Wang et al. 2011; Giljohann et al. 2010; Hasebroock and Serkova 2009). SPIONs can be used as contrasting agent where iron oxides are non-toxic and believed to be metabolized into hemoglobin in body (Laurent et al. 2008; Rosen et al. 2012; Gupta and Gupta, 2005).

\section{Conclusion}

In this study, precursor solution for SPION synthesis was standardized. The produced SPIONs were found to be crystalline and the size was observed between 15 and $20 \mathrm{~nm}$. The SPION was found to be dispersed and spherically shaped which was confirmed by SEM and AFM. The crystallinity pattern confirms the SPION to be $\mathrm{Fe}_{2} \mathrm{O}_{3}$. SPIONs were utilized as contrasting agent imaging quail's egg and it was found to enhance the imaging pattern.

\section{Compliance with ethical standards}

Conflict of interest The authors confirm that there is no conflict of interest.

Funding The work was not funded by any agency.

Open Access This article is distributed under the terms of the Creative Commons Attribution 4.0 International License (http:// creativecommons.org/licenses/by/4.0/), which permits unrestricted use, distribution, and reproduction in any medium, provided you give appropriate credit to the original author(s) and the source, provide a link to the Creative Commons license, and indicate if changes were made.

\section{References}

Bolden NW, Rangari VK, Jeelani S (2008) Synthesis of magnetic nanoparticles and its application in drug delivery systems. In: Laudon M, Romanowicz B (eds) NSTI nanotech, 
nanotechnology conference and trade show, technical proceedings. Boston, MA, United States, June 1-5, pp 390-393

Colombo M, Carregal-Romero S, Casula MF, Gutiérrez L, Morales MP, Böhm IB, Heverhagen JT, Prosperi D, Parak WJ (2012) Biological applications of magnetic nanoparticles. Chem Soc Rev 41:4306-4334

Cortajarena AL, Ortega D, Ocampo SM, Gonzalez-Garcia A, Couleaud P, Miranda R, Belda-Iniesta C, Ayuuso-Sacido A (2014) Engineering Iron oxide nanoparticles for clinical settings. Nanobiomedicine 1(2):1-20

Disch S, Wetterskog E, Hermann RP, Wiedenmann A, Vainio U, Salazar-Alvarez G, Bergstrom L, Bruckl Th (2012) Quantitative spatial magnetization distribution in iron oxide nanocubes and nanospheres by polarized small-angle neutron scattering. New $\mathbf{J}$ Phys 14:013025

Fuller KJ, Issels RD, Slosman DO, Guillet JG, Soussi T, Polla BS (1994) Cancer and the heat-shock response. Eur J Cancer 30A(12):1884-1891

Generalov R, Kuan WB, Chen W, Kristensen S, Juzenas P (2015) Radiosensitizing effect of zinc oxide and silica nanocomposites on cancer cells. Colloids Surf B Biointerfaces 1(129):79-86

Giljohann DA, Seferos DS, Daniel WL, Massich MD, Patel PC, Mirkin CA (2010) Gold nanoparticles for biology and medicine. Angew Chem Int Ed Engl 49(19):3280-3294

Gupta AK, Gupta M (2005) Synthesis and surface engineering of iron oxide nanoparticles for biomedical applications. Biomaterials 26:3995-4021

Harmon BV, Takano YS, Winterford CM, Gobe GC (1991) The role of apoptosis in the response of cells and tumours to mild hyperthermia. Int J Radiat Biol 59(2):489-501

Hasebroock KM, Serkova NJ (2009) Toxicity of MRI and CT contrast agents. Expert Opin Drug Metab Toxicol 5(4):403-416

Jadhav SA, Bongiovanni R (2012) Synthesis and organic functionalization approaches for magnetite $\left(\mathrm{Fe}_{3} \mathrm{O}_{4}\right)$ nanoparticles. $\mathrm{Adv}$ Mat Lett 3(5):356-361

Jalilian AR, Panahifar A, Mahmoudi M, Akhlaghi M, Simchi A (2009) Preparation and biological evaluation of [67 Ga]-labeledsuperparamagnetic nanoparticles in normal rats. Radiochim Acta 97(1):51-56

Kandasamy G, Maity D (2015) Recent advances in superparamagnetic iron oxide nanoparticles (SPIONs) for in vitro and in vivo cancer nanotheranostics. Int J Pharm 496:191-218

Kievit FM, Zhang M (2011) Surface engineering of Iron oxide nanoparticles for targeted cancer therapy. Acc Chem Res 44(10):853-862

Klein S, Sommer A, Distel LV, Hazemann JL, Kroner W, Neuber W, Muller P, Proux O, Kryschi C (2014) Superparamagnetic iron oxide Nanoparticles as X-ray enhancer for low-dose radiation therapy. J Phys Chem B 118(23):6159-6166

Kwatra D, Venugopal A, Anant S (2013) Nanoparticles in radiation therapy: a summary of various approaches to enhance radiosensitization in cancer. Transl Cancer Res 2(4):330-342

Laurent S, Forge D, Port M, Roch A, Robic C et al (2008) Magnetic iron oxide nanoparticles: synthesis, stabilization, vectorization, physicochemical characterizations, and biological applications. Chem Rev 108:2064-2110

Layek S, Pandey A, Pandey A, Verma HC (2010) Synthesis of $\gamma-$ $\mathrm{Fe}_{2} \mathrm{O}_{3}$ nanoparticles with crystallographic and magnetic texture. Int J Eng Sci Technol 2(8):33-39

Leder A, Raschzok N, Schmidt C, Arabacioglu D, Butter A, Kolano S, Lisboa LSDS, Werner W, Polenz D, Reutzel-Selke A, Pratschke J, Sauer IM (2015) Micron-sized iron oxide-containing particles for microRNA-targeted manipulation and MRI-based tracking of transplanted cells. Biomaterials 51:129-137
Lee N, Hyeon T (2012) Designed synthesis of uniformly sized iron oxide nanoparticles for efficient magnetic resonance imaging contrast agents. Chem Soc Rev 41:2575-2589

Li W, Zaloga J, Ding Y, Liu Y, Janko C, Pischetsrieder M, Alexiou C, Boccaccini AR (2016) Facile preparation of multifunctional superparamagnetic PHBV microspheres containing SPIONs for biomedical applications. Sci Rep 6:23140

Martinez-Boubeta C, Simeonidis K, Makridis A, Angelakeris M, Iglesias O, Guardia P, Cabot A, Yedra L, Estradé S, Peiró F, Saghi Z, Midgley PA, Conde-Leborán I, Serantes D, Baldomir D (2013) Learning from nature to improve the heat generation of iron-oxide nanoparticles for magnetic hyperthermia applications. Sci Rep 3:1652

Mitchell E, De Souza F, Gupta RK (2015) Probing on the hydrothermally synthesized iron oxide nanoparticles for ultracapacitor applications. Powder Technol 272:295-299

Mohapatra M, Anand S (2010) Synthesis and applications of nanostructured iron oxides/hydroxides. Int J Eng Sci Technol 12(8):127-146

Nunes JS, de Vasconcelos CL, Cabral FAO, de Araujo JH, Pereira MR, Fonseca JLC (2006) Synthesis and characterization of poly(ethyl methacrylate-co-methacrylic acid) magnetic particles via mini emulsion polymerization. Polymer 47(22):7646-7652

Rand D, Ortiz V, Liu Y, Derdak Z, Wands JR, Taticek M, RosePetruck C (2011) Nanomaterials for X-ray imaging: Gold nanoparticle enhancement of X-ray scatter imaging of hepatocellular carcinoma. Nano Lett 11:2678-2683

Rand D, Walsh EG, Derdak Z, Wands JR, Rose-Petruck C (2015) A highly sensitive X-ray imaging modality for hepatocellular carcinoma detection in vitro. Phys Med Biol 60(2):769-784

Retif P, Pinel S, Toussaint M, Frochot C, Chouikrat R, Thierry Bastogne, Barberi-Heyob M (2015) Nanoparticles for radiation therapy enhancement: the key parameters. Ivyspring Theranost 5(9): 1030-1044

Rosen JE, Chan L, Shieh D-B, Gu FX (2012) Iron oxide nanoparticles for targeted cancer imaging and diagnostics. Nanomed Nanotechnol Biol Med 8:275-290

Salopek B, Krasic D, Filipovic S (1992) Measurement and application of zeta-potential. Rudarsko-geolosko zbornik 4:147-151

Samrot AV, Justin C, Padmanaban S, Burman U (2016) A study on the effect of chemically synthesized magnetite nanoparticles on earthworm: Eudrilus eugeniae. Appl Nanosci 7:17-23

Schleich N, Sibret P, Danhier P, Ucakar B, Laurent S, Muller RN, Jerome C, Gallez B, Preat V, Danhier F (2013) Dual anticancer drug/superparamagnetic iron oxide loaded PLGA-based nanoparticles for cancer therapy and magnetic resonance imaging. Int J Pharm 447:94-101

Schulten K, Weller A (1978) Exploring fast electron transfer processes by magnetic fields. Biophys J 24:295-305

Shubayev VI, Ii PTR, Jin S (2009) Magnetic nanoparticles for theragnostics. Adv Drug Deliv Rev 61(6):467-477

Talelli M, Rijcken CJF, Lammers T, Seevinck PR, Storm G, van Nostrum CF, Hennink WE (2009) Superparamagnetic iron oxide nanoparticles encapsulated in biodegradable thermosensitive polymeric micelles: toward a targeted nanomedicine suitable for image-guided drug delivery. Langmuir 25(4):2060-2067

Thomas R, Park IK, Jeong YY (2013) Magnetic iron oxide nanoparticles for multimodal imaging and therapy of cancer. Int J Mol Sci 14(8):15910-15930

Tombácz E, Toth IY, Neszztor D, Illes E, Hajdu A, Szekeres M, Vekas L (2013) Absorption of organic acids on magnetite nanoparticles, $\mathrm{pH}$-dependent colloidal stability and salt tolerance. Colloids Surf A 435:91-96 
Tombácz E, Szekeres M, Hajdú A, Tóth IY, Bauer RA et al (2014) Colloidal stability of carboxylated iron oxide nanomagnets for biomedical use. Period Polytech Chem 58:3-10

Wang H, Wang HS, Liu ZP (2011) Agents that induce pseudo-allergic reaction. Drug Discov Ther 5(5):211-219
Wang X, Niessner R, Knopp D (2014) Magnetic bead-based colorimetric immunoassay for aflatoxin B1 using gold nanoparticles. Sensors 14(11):21535-21548

Yadav MS (2003) Chemistry of transition metals. Dominant Publishers and Distributors, New Delhi, p 45 